\title{
1 Limited sharing of tick-borne hemoparasites between sympatric wild and 2 domestic ungulates
}

4 RIA R. GHAI ${ }^{1 *}$, MATHEW MUTINDA $^{2}$ and VANESSA O. EZENWA ${ }^{1,3}$

5

$6{ }^{1}$ Odum School of Ecology, University of Georgia, 140 E. Green Street, Athens, GA 30602, USA

$7 \quad{ }^{2}$ Kenya Wildlife Service, PO Box 40241-00100, Nairobi, Kenya

$8 \quad{ }^{3}$ Department of Infectious Diseases, College of Veterinary Medicine, University of Georgia, 501 D.W.

9 Brooks Drive, Athens, GA 30602, USA

10

$13 *$ Correspondence:

14 Address: Odum School of Ecology, 140 E Green Street, Athens, GA 30602

15 Telephone: (706) 542-4819

16 Email: riaghai@uga.edu

17

18

19 Running Title: Hemoparasite infections in wild and domestic ruminants

20

21

22

23

24

25 


\section{HIGHLIGHTS}

27 - $99 \%$ of wild Grant's gazelle, $66 \%$ of domestic sheep were PCR-positive for Theileria

- $32 \%$ of Grant's gazelle, $47 \%$ of sheep were PCR-positive for Anaplasma

29

- Theileria sequences sorted into 2 genotypes; each was specific to the host

30

- Anaplasma sequences sorted into 5 genotypes; 1 contained samples from both hosts

31

32

33

34

35

36

37

38

39

40

41

42

43

44

45

46

47

48 


\section{ABSTRACT}

Tick-borne hemoparasites (TBHs) are a group of pathogens of concern in animal management because

51 they are associated with a diversity of hosts, including both wild and domestic species. However, little is

52 known about how frequently TBHs are shared across the wildlife-livestock interface in natural settings.

53 Here, we compared the TBHs of wild Grant's gazelle (Nanger granti) and domestic sheep (Ovis aries)

54 in a region of Kenya where these species extensively overlap. Blood samples collected from each species were screened for piroplasm and rickettsial TBHs by PCR-based amplification of 18S/16S ribosomal DNA, respectively. Overall, $99 \%$ of gazelle and $66 \%$ of sheep were positive for

57

58

59

60

61

62

63

64

65 66

67

68

69

70

71

72 2

Babesia/Theileria, and 32\% of gazelle and 47\% sheep were positive for Anaplasma/Ehrlichia.

Sequencing a subset of positive samples revealed infections of Theileria and Anaplasma. Sequences sorted into seven phylogenetically distinct genotypes - two Theileria, and five Anaplasma. With the exception of a putatively novel Anaplasma lineage from Grant's gazelle, these genotypes appeared to be divergent forms of previously described species, including T. ovis, A. ovis, A. bovis, and A. platys. Only one genotype, which clustered within the A. platys clade, contained sequences from both gazelle and sheep. This suggests that despite niche, habitat, and phylogenetic overlap, the majority of circulating tick-borne diseases may not be shared between these two focal species. Keywords: Anaplasma; Theileria; hemoparasite; tick-borne diseases; parasite sharing; sheep; gazelle 


\section{INTRODUCTION}

74 Pathogen transmission occurring between wild and domestic animals is gaining attention, in part

75 because pathogen spillover can be devastating to both wildlife and livestock (Siembieda et al., 2011;

76 Wiethoelter et al., 2015). For example, wildlife-endemic diseases like foot-and-mouth disease and

77 heartwater that naturally occur in African buffalo (Syncerus caffer) are now considered among the most

78 serious livestock diseases on the African continent, particularly in cattle (Bengis et al., 2002). On the

79 other hand, livestock diseases like rinderpest and brucellosis have contributed to precipitous wild

80

81

82

83

84

85

86

87

88

89

90

91

92

93

94

95 ruminant declines in Africa and North America, (Grootenhuis, 2000; Miller et al., 2013; Nishi et al., 2002). Given that livestock and wildlife are estimated to share three quarters of their pathogens (Cleaveland et al., 2001; Wiethoelter et al., 2015), spillover events between the two groups may be inevitable. Nevertheless, understanding the ease with which various pathogen taxa transmit across the wildlife-livestock interface may help narrow the scope of efforts seeking to prevent future outbreaks.

Tick-borne hemoparasites (TBHs) are one group of pathogens that occur in both livestock and wildlife, and may be commonly transmitted between wild and domestic species (Dantas-Torres et al., 2012; Gortazar et al., 2007). Not only do TBHs cause significant morbidity and mortality in livestock (Uilenberg, 1995), but wildlife can contribute to livestock TBHs by acting as both sources and maintenance hosts for disease (Kock, 2005). For example, East Coast Fever, caused by the protozoan parasite Theileria parva, originates from African buffalo which harbor "silent" infections, and now circulates in cattle, which often succumb to the disease (Bengis et al., 2002; Olwoch et al., 2008). Similarly, free-ranging white-tailed deer (Odocoileus virginianus) and sika deer (Cervus nippon) have been identified as reservoir hosts that maintain transmission of piroplasm (Babesia bigemina, B. ovis) and rickettsial (Anaplasma bovis, A. centrale, A. phagocytophilum, and Ehrlichia spp.) TBHs endemic 
to livestock in Mexico and Japan, respectively (Cantu et al., 2007; Kawahara et al., 2006). Livestock

97 TBHs may also impact wildlife (Miller et al., 2013), although TBH spillover from livestock to wildlife

One way to understand the likelihood of pathogen spillover is to quantify the frequency with which wildlife and livestock share pathogens under natural conditions. Increased TBH sharing between wildlife and livestock may occur for at least three reasons. First, interactions between wildlife and livestock are expanding due to human-induced changes (Miller et al., 2013; Wiethoelter et al., 2015). As such, increases in direct or indirect contact (e.g. habitat overlap) may facilitate exposure and sharing of previously isolated pathogens (Daszak et al., 2001; Maxwell et al., 2013). Human translocation of wildlife or livestock to new areas is one possible means of increasing contact between wild and domestic species. Translocations have resulted in outbreaks and mortality among naïve inhabitants—as was the case in cattle when Theileria parva-infected buffalo were translocated to the Highveld of Zimbabwe (Latif et al., 2002). Second, many TBHs are vectored by ticks that have broad host ranges and require multiple blood meals to complete their lifecycles (Jongejan and Uilenberg, 2004). Pathogens vectored by ticks with catholic feeding habits are more likely to be shared between species, because these vectors can facilitate exposure to new hosts with each feeding event (McCoy et al., 2013; Shaw et al., 2001).

Theileria parva is a striking example of this, being vectored primarily by the brown ear tick (Rhipicephalus appendiculatus), which requires three separate hosts to complete development. Interestingly, this tick has been collected from over a hundred host species (Cumming, 1998; Jongejan and Uilenberg, 2004), which may contribute to T. parva's propensity for transmission across the wildlife-livestock interface. Finally, hosts that are phylogenetically closely related are often more likely to share pathogens than distantly related hosts, due to physiological and ecological similarity (Davies 
and Pedersen, 2008). For example, a recent study of 16 TBH species circulating among 18 wild and domestic ungulates in South Africa found that TBHs tended to cluster by the phylogenetic history of

121 hosts (Berggoetz et al., 2014).

On the African continent, TBHs of greatest concern include protozoan piroplasms of the genera Babesia and Theileria, and rickettsial bacteria of the genera Anaplasma and Ehrlichia. These species are common in cattle, sheep, and goats (Njiiri et al., 2015; Uilenberg, 1995), and are an increasingly reported in wildlife (Criado-Fornelio et al., 2004; Eygelaar et al., 2015; Heyman et al., 2010). However, descriptions of TBH sharing between wildlife and livestock are infrequent. Here, we examine the extent to which these TBHs are shared between a wild (Grant's gazelle) and domestic (sheep) ruminant in central Kenya. In this region, livestock densities are increasing, and there is extensive overlap between livestock and wildlife populations (Georgiadis et al., 2007; Kinnaird and O'Brien, 2012). Interestingly, a close relative of Theileria ovis (a common TBH of sheep; Altay et al. (2005)), was recently isolated from Grant's gazelle (Nanger granti) (Hooge et al. 2015). This raises the question of whether this new genotype is transmissible to domestic animals, which extensively overlap with wildlife in the study region. Grant's gazelle occur across East Africa, can persist at high livestock density (Georgiadis et al., 2007), and also host a number of tick species known to infest livestock (Walker et al., 2003). Using molecular detection by PCR and sequencing, we screened Grant's gazelle samples collected in three separate years for the piroplasms Babesia and Theileria, and the rickettsiae Anaplasma and Ehrlichia.

We compared these TBH profiles to those from sheep inhabiting high wildlife density (considerable wildlife-livestock overlap) and low wildlife density (little wildlife-livestock overlap) areas. This 
141 gazelle, and 2) whether these genotypes were more likely to be shared under conditions of greater host 142 overlap.

\section{METHODS}

\subsection{Animal sampling}

146 This research was approved by the University of Georgia Animal Care and Use Committee (\#A2013 08147 018-Y3-A1 and \#A2015 04-004-Y1-A0). All samples were collected in Laikipia County, Kenya.

148 Grant's gazelle were sampled at the Mpala Research Center (MRC) in August 2009 (n = 62), July 2011 $149(\mathrm{n}=62)$, and June $2015(\mathrm{n}=58)$. The total samples size $(\mathrm{n}=182)$ represents $40-60 \%$ of the total gazelle 150 population at MRC during the sampling period. Gazelle were captured by helicopter using a hand-held 151 net gun fired from the aircraft. Sheep were sampled at two locations: MRC $(\mathrm{n}=50)$, a private ranch with 152 low-intensity livestock production and considerable wildlife-livestock overlap, and Lekiji $(\mathrm{n}=84)$, an 153 adjacent community-owned ranch with higher intensity livestock production and significantly less 154 wildlife-livestock overlap (Georgiadis et al., 2007). Sheep sampling occurred between June 8 and July 1 155 2015. Samples from MRC were collected from a single herd of 92 animals, while samples from Lekiji 156 were collected from eight herds that varied in size from 17-112 animals. Owner consent was granted 157 prior to all sampling.

159 For all animals, blood was collected from the jugular vein into $10 \mathrm{~mL}$ heparinized vacutainer tubes as 160 described in Ezenwa et al. (2012). In addition to blood sampling, we also collected information on the 161 number of days since the last acaricide treatment for sheep, since we considered that regular treatment 162 could diminish the prevalence and therefore detection rate of TBHs in this species. All blood samples 
163 were kept on ice in the field until transport to the laboratory where they were stored at $-20^{\circ} \mathrm{C}$ until 164 processing.

$166 \quad 2.2$ Parasitological analyses

167 DNA was extracted from $100 \mu \mathrm{L}$ of whole blood using the Qiagen DNeasy Blood and Tissue Kit 168 (Qiagen, CA, USA) according to the manufacturer's instructions. To screen for Babesia/Theileria, we 169 followed the polymerase chain reaction (PCR) protocol (reagents and cycling parameters) used by 170 Hooge et al. (2015). This method amplified a $\sim 40 \mathrm{bp}$ fragment of the V4 hypervariable region of 18S 171 ribosomal DNA. Specifically, primers RLB F2 (5'-GAC ACA GGG AGG TAG TGA CAA G-3') and 172 RLB R2 (5'-CTA AGA ATT TCA CCT CTA ACA GT-3') were used (Gubbels et al., 1999). The results 173 of Babesia/Theileria infection from the 2009 Grant's gazelle sample set were recently published (Hooge 174 et al., 2015); here, we screened gazelle samples from 2011 and 2015 and all sheep samples (2015, MRC 175 and Lekiji).

176

177 To screen for Anaplasma/Ehrlichia, we amplified a $\sim 450 \mathrm{bp}$ fragment of the V1 hypervariable region of 178 16S ribosomal DNA using previously published primers EHR-F (5'-GGA ATT CAG AGT TGG ATC 179 MTG GYT CAG-3') and EHR-R (5'-CGG GAT CCC GAG TTT GCC GGG ACT TYT TCT-3') 180 (Bekker et al., 2002). This reaction was carried out in $25 \mu \mathrm{L}$ volumes. The reaction mixture contained 3 $181 \mu \mathrm{l}$ of template DNA, $0.4 \mu \mathrm{M}$ of each forward and reverse primer, $200 \mu \mathrm{M}$ of dNTP, $2.5 \mu \mathrm{L} 10 \mathrm{X}$ PCR 182 buffer, $2.0 \mathrm{mM}$ of $\mathrm{MgCl}_{2}, 5 \mu \mathrm{L} 360$ GC Enhancer, and $0.25 \mu \mathrm{L}$ (1.25 units) of AmpliTaq Gold 360 DNA 183 Polymerase (all reagents from Thermo Fisher Scientific, NY, CA, USA). Reactions were cycled with the 184 following thermal profile: $95^{\circ} \mathrm{C}$ for $10 \mathrm{~min} ., 35$ cycles of $95^{\circ} \mathrm{C}$ for $30 \mathrm{sec} ., 55.5^{\circ} \mathrm{C}$ for $30 \mathrm{sec}$, and $72^{\circ} \mathrm{C}$ 
185 for $60 \mathrm{sec}$, and a final $10 \mathrm{~min}$. extension at $72^{\circ} \mathrm{C}$. All gazelle samples $(2009,2011$ and 2015$)$, and all 186 sheep samples (2015 MRC and Lekiji) were screened by this method.

188 All PCR products were electrophoresed on a 1\% agarose gel stained with GelRed (Biotium Inc., CA, 189 USA) and visualized under UV light to determine positivity for Babesia/Theileria and 190 Anaplasma/Ehrlichia. A subset of samples with the most brightly banded PCR products were selected 191 for sequencing. A total of 38 gazelle samples $(n=18$ from 2015, 20 from 2011) and 36 sheep samples (n $192=18$ from MRC, 18 from Lekiji) were sequenced for Babesia/Theileria. In addition, 21 gazelle samples $193(\mathrm{n}=1$ from 2015,13 from 2011, 7 from 2009) and 34 sheep samples ( $\mathrm{n}=7$ from MRC, 27 from Lekiji) 194 were sequenced for Anaplasma/Ehrlichia. Selected samples were purified using the QIAquick PCR 195 Purification Kit (Qiagen, CA, USA) and Sanger sequenced on an ABI3730 Genetic Analyzer (Applied 196 Biosystems, CA, USA) using both forward and reverse primers. Sequencing results were edited using 197 Geneious v. 8.1 (Biomatters, Auckland, New Zealand).

\subsection{Statistical Analyses}

200 To test for effects of acaricide treatment on TBH infection status in sheep, we used generalized linear 201 mixed models (GLMMs) with a binomial error distribution. Two separate models were run with either 202 Theileria or Anaplasma infection status as the response variable. Infection status was classified as a 203 binary variable (either uninfected or infected). The main predictor variable was time since last acaricide 204 treatment; age, sex, and location (MRC or Lekiji) were included as covariates. Herd membership was 205 also included as a random effect. Models were run in R v. 3.2.0 (R-Development-Team, 2015). 206 
Theileria and Anaplasma sequences were separately aligned in ClustalX v. 2.1. Theileria sequences isolated from gazelle and sheep in this study were aligned with 76 representative Theileria sequences (including the GG1, GG2 and GG3 genotypes identified by Hooge et al. [2015] from 2009 gazelle) and a Toxoplasma gondii outgroup sequence. Anaplasma sequences isolated in this work were aligned with 29 representative Anaplasma sequences and an Ehrlichia ruminatum outgroup sequence. All representative sequences used were available from Genbank (see S4), and were required to fit two of three criteria for inclusion: 1) belonging to scientific papers published in peer-reviewed literature; 2) isolated from African mammals; 3) isolated from ungulates (both Artiodactyla and Perrisodactyla). For both alignments, poorly aligned regions were manually adjusted and regions of ambiguous alignment were identified and removed in Gblocks v. 0.91 using the least stringent parameter settings (Castresana, 2000; Talavera and Castresana, 2007). Alignments were trimmed to identical lengths and models of sequence evolution were selected using the MrModelTest v. 2.3 executable in PAUP* v. 4 (Nylander, 2004; Swofford, 2003).

Phylogenetic relationships were reconstructed using maximum likelihood methods in PhyML v. 3.0 (Guindon et al., 2010; Guindon and Gascuel, 2003). Specifically, the Tamura-Nei model with gamma shape parameter and fixed (invariant) sites was selected for Theileria (Tamura and Nei, 1993), while the Kimura 2-parameter model with a gamma shape parameter and fixed sites (Kimura, 1980) was selected for Anaplasma. Phylogeny support was assessed using 1000 bootstrap iterations. Percentage nucleotide similarity within and between clades was calculated for both Theileria and Anaplasma using 1000 bootstrap replicates of the uncorrected pairwise proportion of divergent nucleotides ( $p$-distance) in MEGA v. 6.0 (Tamura et al., 2013). Sequence data reported in this paper are available in GenBank under accession numbers KU569699 (Theileria) and KU569700-4 (Anaplasma). 


\section{RESULTS}

\subsection{Patterns of Infection}

\subsubsection{Babesia/Theileria}

235 A total of 120 Grant's gazelle samples from $2011(\mathrm{n}=62)$ and $2015(\mathrm{n}=58)$, and 134 sheep samples 236 from MRC $(\mathrm{n}=50)$ and Lekiji $(\mathrm{n}=84)$ were screened for Babesisa/Theileria (Table 1). In gazelle, a 23798.4 and 100\% prevalence of Babesia/Theileria was detected in 2011 and 2015, respectively, which is 238 similar to the $100 \%$ prevalence documented by Hooge et al (2015) for animals sampled in $2009(\mathrm{n}=65)$. 239 In sheep, 58\% of animals sampled at MRC and 71.4\% of animals sampled at Lekiji were 240 Babesia/Theileria positive (Table 1). There was no association between time since the last acaricide 241 treatment and Babesia/Theileria infection status (Number days since acaricide treatment: $Z=-0.52, P=$ 242 0.603; Age: $Z=1.60, P=0.111$; Location (MRC): $Z=-2.44, P=0.015$; Sex (Male): $Z=1.97, P=$ 2430.049 ), so it seems unlikely that treatment affected our ability to detect this parasite in sheep. All 244 sequenced samples from both gazelle and sheep were identified as Theileria spp., which suggests that 245 infections by Babesia were absent.

\subsubsection{Anaplasma/Ehrlichia}

248 A total of 179 Grant's gazelle samples from $2009(n=59), 2011$ and 2015 ( $n$ as above) and 134 sheep 249 samples from MRC and Lekiji (n as above) were screened for Anaplasma/Ehrlichia. Grant's gazelle had 250 a prevalence of 52.5, 41.9, and $1.7 \%$ in 2009,2011 , and 2015, respectively. In sheep, $14.0 \%$ of animals 251 sampled at MRC and 66.7\% sampled at Lekiji were positive for Anaplasma/Ehrlichia. As with

252 Theileria, there was no relationship between time since last acaricide treatment and 253 Anaplasma/Ehrlichia prevalence in sheep (Number days since acaricide treatment: $Z=1.001, P=0.314$; 
254 Age: $Z=1.95, P=0.051$; Location (MRC): $Z=-3.73, P=<0.001$; Sex (Male): $Z=-1.61, P=0.108)$.

255 All sequenced samples from both gazelle and sheep were identified as Anaplasma spp., suggesting that 256 infections by Ehrlichia were absent.

3.2. Phylogenetic Relationships

259 3.2.1. Theileria

260 From 38 Grant's gazelle and 36 sheep samples sequenced, 14 gazelle and seven sheep sequences had 261 conspicuous overlapping chromatogram peaks that were suggestive of co-infection, as has been found 262 previously in Grant's gazelle (Hooge et al., 2015). Another five sheep sequences were of poor quality. 263 These 26 sequences were omitted from phylogenetic analyses. The remaining 48 sequences from gazelle 264 (2011: $\mathrm{n}=11 \& 2015 \mathrm{n}=13)$ and sheep (MRC: $\mathrm{n}=11$ \& Lekiji: $\mathrm{n}=13)$ were used for phylogenetic 265 reconstruction. These analyses revealed two distinct Theileria genotypes that were specific to either 266 sheep or gazelle (Fig. 1). Within these genotypes, all sequences were $100 \%$ identical. The sheep 267 genotype, designated ThSh1 (for Theileria Sheep Genotype 1), contained all 24 sheep sequences, and 268 was $99.9 \%$ identical to representative $T$. ovis sequences. The gazelle genotype contained all 24 gazelle 269 sequences and was $100 \%$ identical to GG2, a genotype previously described by Hooge et al. (2015) (Fig. 270 2). Interestingly, GG2 was the only genotype isolated from 2011 and 2015 gazelle samples, although 271 Hooge et al. (2015) isolated GG1 and GG3 in addition to GG2 from 2009 samples. The GG2 genotype 272 differed from GG1 by a single nucleotide substitution, and shared 94.5\% nucleotide identity with GG3. 273 GG2 shared 99.4\% sequence identity with T. ovis, and appears to be sister to the clade containing both 274 T. ovis and the newly identified sheep genotype ThSh1. 
276 The sheep (ThSh1) and gazelle (GG2) genotypes identified in this study were 99.4\% identical. These

277 genotypes differed by five nucleotides that were consistent based on host isolation source (gazelle or 278 sheep). ThSh1 shared 99.4 and $93.7 \%$ nucleotide similarity with GG1 and GG3, the two gazelle 279 genotypes reported from Hooge et al (2009). Both sheep and gazelle genotypes were most divergent 280 from T. equi ( $87 \%$ sequence similarity), and shared $76 \%$ similarity with the outgroup Toxoplasma gondii 281 (see S1 for all pairwise distance calculations).

\subsubsection{Anaplasma}

284 Out of 21 Grant's gazelle and 34 sheep samples sequenced, 19 gazelle and 25 sheep samples returned usable sequence data. Two gazelle and six sheep samples had conspicuous overlapping chromatogram peaks suggestive of co-infection. This included the only gazelle sample positive for Anaplasma in 2015.

287 An additional three sheep sequences were of poor quality. All 11 of these sequences were omitted from 288 further analyses. The remaining 44 sequences from gazelle (2009: $\mathrm{n}=7 \& 2011: \mathrm{n}=12)$ and sheep (MRC: $\mathrm{n}=3$ \& Lekiji: $\mathrm{n}=22$ ) were used for phylogenetic reconstruction. These analyses revealed that the Anaplasma genotypes detected in this study sorted into five distinct clades. These clades were designated AnSh1, AnGa2, AnSh3, AnGa4, and $\mathrm{AnGa}+\mathrm{Sh} 5$ to represent the genus (An for "Anaplasma"), host species the sample was isolated from (Ga for "gazelle" or Sh for "sheep"), and an arbitrary numeric value of 1-5 to represent the five clades discovered here. Genotypes 1 and 3 were only 294 isolated from sheep, and genotypes 2 and 4 were only isolated from gazelle. Genotype 5 was isolated 295 from both sheep and gazelle. The first genotype, AnSh1, comprised $56 \%$ of all successfully sequenced 296 sheep samples. AnSh1 formed a monophyletic clade that was sister to AnGa2 and AnSh3, and 99.7\% 297 sequence similarity, respectively. AnGa2 comprised $16 \%$ of sequenced gazelle samples, and shared 
genotypes all differed from one another by three nucleotide substitutions, and formed a monophyletic 300 sister group to representative A. ovis sequences. AnSh1, AnGa2 and AnSh3 shared 99.5, 99.0, and $99.3 \%$ sequence similarity with the $A$. ovis clade, respectively. AnGa4 comprised $63 \%$ of all sequenced gazelle samples. AnGa4 clustered with the A. bovis clade and shared $99.1 \%$ sequence identity with other

marginale, A. ovis, and AnSh1, AnGa2, and AnSh3. The gazelle genotype AnGa4 shared greater sequence similarity (96.7\%) with the gazelle genotype AnGa2, than with the sheep genotypes AnSh1 and AnSh3 (96.1\%). Finally, AnGa+Sh5 sequences were isolated from both Grant's gazelle and sheep. $25 \%$ of successfully sequenced gazelle samples in 2009 and $14 \%$ in 2011 were infected with the AnGa+Sh5 genotype, compared with 9\% of sheep from Lekiji and none from MRC. This genotype was the most divergent of those isolated in this study, sharing between 95.2 (AnSh1, AnGa2, AnSh3) and 97.2\% (AnGa4) sequence similarity with the other genotypes isolated here. AnGa+Sh5 sequences fell within the A. platys species complex and shared $99.3 \%$ sequence identity with other $A$. platys sequences (see S2 for all pairwise distance calculations).

\section{DISCUSSION}

This study investigated the potential for $\mathrm{TBH}$ sharing between one wild and one domestic ruminant in central Kenya. Based on habitat overlap, shared tick vectors, and family-level phylogenetic relatedness, we expected that Grant's gazelle and sheep would share some of their TBHs. We discovered seven distinct TBH genotypes (two Theileria spp., and five Anaplasma spp.), and only one (Anaplasma: $\mathrm{AnGa}+\mathrm{Sh} 5)$ was identical in both gazelle and sheep. Interestingly, this genotype was only detected in sheep at the high-intensity livestock site where overlap with wildlife (including gazelle) is limited 
321 (Georgiadis et al., 2007). These results suggest that despite an opportune system for pathogen sharing, 322 the majority of TBH infections are not shared across the wildlife-livestock interface in this system.

Although there was one identical TBH genotype (AnGa+Sh5) in sheep and gazelle, this was the most uncommon Anaplasma genotype in sheep, isolated from only $8 \%$ of sequenced samples (compared to AnSh1 [56\%] and AnSh3 [36\%]). AnGa+Sh5 was also rare in gazelles, isolated from 21\% of sequenced 327 gazelle samples (compared to AnGa2 [16\%] and AnGa4 [63\%]). Interestingly, this genotype clustered with A. platys sequences and may therefore be a divergent member of this clade. A. platys is typically considered an exclusive canine parasite that causes infectious canine cyclic thrombocytopenia, a disease associated with low platelet counts in dogs (Harvey et al., 1978; Yabsley et al., 2008). However, other recent studies have identified $A$. platys-like sequences in ruminants, including cattle and goats in China 332 (Liu et al., 2012; Yang et al., 2015), and sheep from Sardinia and South Africa (Berggoetz et al., 2014; Zobba et al., 2014). Given our discovery of an A. platys-like genotype in both a wild ruminant and 334 domestic ruminant in Kenya, future research to determine whether ruminants can act as alternative hosts for A. platys (as suggested by Yang et al. (2015)) or whether they host phylogenetically similar lineages with distinct host ranges seems warranted.

The majority of genotypes isolated in this study differed between gazelle and sheep by five or fewer nucleotides (Theileria: ThSh1 and GG2; Anaplasma: AnSh1, AnGa2, AnSh3; see S3 for alignments). 340 While these substitutions alone may not preclude transmissibility between hosts, the conservation of 341 these nucleotide differences between hosts suggests that Grant's gazelle genotypes are not necessarily 342 readily transmissible to sheep, or vice versa. This notion of minor genetic host specificity corroborates 343 results of previous research by de la Fuente et al. (2007), which found that genotypes of A. ovis from 
344 wild mule deer (Odocoileus hemionus) and bighorn sheep (Ovis canadensis) were distinct by between

345 one and three nucleotides from previously characterized genotypes of domestic sheep. Similarly,

346 research in central Spain found that while overlapping populations of Iberian red deer (Cervus elaphus

347 hispanicus) and domestic cattle both harbored Anaplasma marginale and A. phagocytophilum, each

348 species possessed distinct genotypes that were $>95 \%$ genetically similar to one another (De La Fuente et

349 al., 2005). Taken together, it seems possible that these host-specific genotypes are less readily

350 transmissible across the wildlife-livestock interface. Nevertheless, wildlife and livestock co-habitation

351 may still influence TBD dynamics by contributing to the maintenance of generalist tick populations.

353 Aside from the examples above, studies that compare the TBHs of wild and domestic species occurring 354 in the same geographic area are rare. One notable exception characterized the infections of 18 wild and 355 domestic hosts, which harbored 16 TBHs of the genera Babesia, Theileria, Anaplasma and Ehrlichia 356 (Berggoetz et al., 2014). The study described broader host ranges than previously reported for several 357 TBHs, including multiple Theileria (T. bicornis, T. buffeli, T. separata, T. sp) and Anaplasma (A. 358 centrale, marginale) species (Berggoetz et al., 2014). This appears contrary to our results of subtle 359 nucleotide specificity between host species. However, Berggoetz and colleagues used reverse line blot 360 (RLB) hybridization to detect species or genus-level TBH infection. The RLB approach is a widely used 361 method for TBH detection (Bekker et al., 2002; Gubbels et al., 1999; Schnittger et al., 2003), and has the 362 advantage of being able to detect multiple species even among co-infected samples. Indeed, inability to 363 use co-infected samples is a limitation of this study. However, PCR-based amplification and sequencing 364 in this study allowed the detection of fine-scale genetic differences that may not be distinguishable with 365 RLB. Future research attempting to quantify the actual cross-species transmission potential of closely 366 related TBH genotypes is needed to better understand the natural host range of these pathogens. 
368 In addition to determining the extent of TBH sharing occurring between wildlife and livestock, we also 369 identified several novel TBH genotypes. For example, finding additional gazelle sequences that cluster 370 with Theileria GG2 supports the conclusions of Hooge et al. (2015) that this genotype, along with GG1, 371 form an uncharacterized clade which should be further investigated for putative species designation. In 372 addition, no other genotype identified was 100\% identical to previously characterized sequences.

373 Discovery of novel genotypes is a recurring pattern in studies that generate TBH sequence data, 374 especially in wildlife. For example, a study of Mongolian gazelle (Procapra gutturosa) in China 375 detected four Anaplasma species (A. bovis, A. ovis, A. phagocytophilum, and an uncharacterized lineage) 376 and several divergent forms of Theileria luwenshuni (Li et al., 2014). Similarly, several new genotypes 377 of Theileria have been identified in waterbuck (Kobus defassa) and African buffalo from Kenya (Chaisi 378 et al., 2013; Githaka et al., 2014). Taken together, these results suggest that there remains significant 379 uncharacterized diversity within TBHs.

\section{CONCLUSIONS}

382 This study discovered new genotypes of both Theileria and Anaplasma in domestic sheep and wild 383 Grant's gazelle, which supports a common pattern of substantial genetic diversity within TBHs. Perhaps 384 more intriguingly, however, we discovered that despite opportune conditions for transmission across the 385 wildlife-livestock interface, there was limited evidence of TBH sharing between this spatially 386 overlapping pair of wild and domestic small ruminant. Although considerable attention has focused on 387 pathogens that transmit across host species, the frequency of tick-borne hemoparasite sharing we 388 document here suggests that transmission between livestock and wildlife may be less common than is 389 widely expected. 
CONFLICT OF INTEREST

392 The authors declare that there are no conflicts of interest.

394 ACKNOWLEDGEMENTS

395 Permission to conduct this research was granted by the Kenyan National Commission for Science, 396 Technology and Innovation and the Kenya Wildlife Service. Grant's gazelle captures were conducted by 397 Frontier Helicopters, NZ, and the Kenya Wildlife Service Game Capture Unit. We thank John Ewoi, 398 Sara Heisel, Mike Littlewood, Aaron Onserio, Mireya Smith, and Allison Williams for assistance in the 399 field; the Mpala Research Center for logistical support; and Christopher Cleveland and Michael Yabsley 400 for providing positive controls and protocols. The study was funded by a National Science Foundation 401 grant (IOS-1101836) to VOE. RRG was supported by a Fonds de Recherche du Quebec - Nature et 402 Technologies postdoctoral fellowship.

403 404 405 406 407 408 409 410 411 


\section{TABLES}

416 Table 1. Prevalence of TBHs Theileria and Anaplasma based on PCR and sequencing. " $n$ " indicates the 417 total number of samples collected.

418

419

420

421

\begin{tabular}{|c|c|c|c|c|c|c|c|}
\hline & \multicolumn{4}{|c|}{ Grant's Gazelle - MRC* } & \multicolumn{3}{|c|}{ Sheep - 2015} \\
\hline & $\begin{array}{l}2015 \\
(n=58)\end{array}$ & $\begin{array}{l}2011 \\
(\mathrm{n}=62)\end{array}$ & $\begin{array}{l}2009 \\
(n=59)\end{array}$ & $\begin{array}{l}\text { TOTAL } \\
(\mathrm{n}=179)\end{array}$ & $\begin{array}{l}\mathrm{MRC} \\
(\mathrm{n}=50)\end{array}$ & $\begin{array}{l}\text { Lekiji } \\
(\mathrm{n}=84)\end{array}$ & $\begin{array}{l}\text { TOTAL } \\
(\mathrm{n}=134)\end{array}$ \\
\hline Theileria & 100.0 & 98.4 & 100.0 & 99.4 & 58.0 & 71.4 & 66.4 \\
\hline Anaplasma & 1.7 & 41.9 & 52.5 & 32.4 & 14.0 & 66.7 & 47.0 \\
\hline
\end{tabular}

*MRC $=$ Mpala Research Center 


\section{FIGURES}

443 Fig. 1. Maximum likelihood (TN93 G + I) phylogenetic reconstruction of Theileria. Sequences isolated 444 from this work shown in bold and black, while sequences isolated from 2009 gazelle from the same 445 population (Hooge et al. 2015) are shown in bold and grey. Square brackets indicate either the number 446 of sequences, or the Genbank accession number if only one or two sequences are represented. See S4 for 447 Genbank accession numbers of all sequences used. Where possible, round brackets were also included to 448 indicate the hosts that the sequences were isolated from. Numeric values at the nodes indicate bootstrap 449 support established through 1000 permutations. Scale bar indicates nucleotide substitutions per site.

Fig. 2. Maximum likelihood (K2 G + I) phylogenetic reconstruction of Anaplasma, with sequences 452 isolated from this work shown in bold. Square brackets indicate either the number of sequences, or the 453 Genbank accession number if only one or two sequences are represented. See S4 for Genbank accession 454 numbers of all sequences used. Where possible, round brackets were also included to indicate the hosts 455 that the sequences were isolated from. Numeric values at the nodes indicate bootstrap support 456 established through 1000 permutations. Scale bar indicates nucleotide substitutions per site. 


\section{REFERENCES}

Altay, K., Dumanli, N., Holman, P.J., Aktas, M., 2005. Detection of Theileria ovis in naturally infected sheep by nested PCR. Vet. Parasitol. 127, 99-104.

Bekker, C.P.J., de Vos, S., Taoufik, A., Sparagano, O.A.E., Jongejan, F., 2002. Simultaneous detection of Anaplasma and Ehrlichia species in ruminants and detection of Ehrlichia ruminantum in Amblyomma variegatum ticks by reverse line blot hybridization. Vet. Microbiol. 89, 223-238.

Bengis, R., Kock, R., Fischer, J., 2002. Infectious animal diseases: the wildlife/livestock interface. Rev. - Off. Int. Epizoot. 21, 53-66.

Berggoetz, M., Schmid, M., Ston, D., Wyss, V., Chevillon, C., Pretorius, A.M., Gern, L., 2014. Tickborne pathogens in the blood of wild and domestic ungulates in South Africa: interplay of game and livestock. Ticks Tick Borns Dis. 5, 166-175.

Cantu, A., Ortega-S, J.A., Mosqueda, J., Garcia-Vazquez, Z., Henke, S.E., George, J.E., 2007. Immunologic and molecular identification of Babesia bovis and Babesia bigemina in freeranging white-tailed deer in Northern Mexico. J. Wildl. Dis. 43, 504-507.

Castresana, J., 2000. Selection of conserved blocks from multiple alignments for their use in phylogenetic analysis. Mol. Biol. Evol. 17, 540-552.

Chaisi, M.E., Collins, N.E., Potgieter, F.T., Oosthuizen, M.C., 2013. Sequence variation identified in the 18S rRNA gene of Theileria mutans and Theileria velifera from the African buffalo (Syncerus caffer). Vet. Parasitol. 191, 132-137. 
Cleaveland, S., Laurenson, M.K., Taylor, L.H., 2001. Diseases of humans and their domestic mammals: pathogen characteristics, host range and the risk of emergence. Philos. Trans. R. Soc. Lond., B, Biol. Sci. 356, 991-999.

Criado-Fornelio, A., Gónzalez del, R., amp, x, o, M.A., Buling-Saraña, A., Barba-Carretero, J.C., 2004. The "expanding universe" of piroplasms. Vet. Parasitol. 119, 337-345.

Cumming, G.S., 1998. Host preference in African ticks (Acari: Ixodida): a quantitative data set. Bull. Entomol. Res. 88, 379-406.

Dantas-Torres, F., Chomel, B.B., Otranto, D., 2012. Ticks and tick-borne diseases: a One Health perspective. Trends Parasitol. 28, 437-446.

Daszak, P., Cunningham, A.A., Hyatt, A.D., 2001. Anthropogenic environmental change and the emergence of infectious diseases in wildlife. Acta Trop. 78, 103-116.

Davies, J.T., Pedersen, A.B., 2008. Phylogeny and geography predict pathogen community similarity in wild primates and humans. Proc. R. Soc. Lond., B, Biol. Sci. 275, 1695-1701.

de la Fuente, J., Atkinson, M.W., Naranjo, V., Fernández de Mera, I.G., Mangold, A.J., Keating, K.A., Kocan, K.M., 2007. Sequence analysis of the msp4 gene of Anaplasma ovis strains. Vet. Microbiol. 119, 375-381.

De La Fuente, J., Naranjo, V., Ruiz-Fons, F., Höfle, U., Fernández De Mera, I.G., Villanúa, D., Almazán, C., Torina, A., Caracappa, S., Kocan, K.M., Gortázar, C., 2005. Potential vertebrate reservoir hosts and invertebrate vectors of Anaplasma marginale and A. phagocytophilum in Central Spain. Vector Borne Zoonotic Dis. 5, 390-401.

Eygelaar, D., Jori, F., Mokopasetso, M., Sibeko, K.P., Collins, N.E., Vorster, I., Troskie, M., Oosthuizen, M.C., 2015. Tick-borne haemoparasites in African buffalo (Syncerus caffer) from two wildlife areas in Northern Botswana. Parasit Vectors 8, 1-11. 
507 Ezenwa, V.O., Stefan Ekernas, L., Creel, S., 2012. Unravelling complex associations between testosterone and parasite infection in the wild. Funct. Ecol. 26, 123-133.

509 Georgiadis, N.J., Olwero, J.G.N., Ojwang', G., Romañach, S.S., 2007. Savanna herbivore dynamics in a 510 livestock-dominated landscape: I. Dependence on land use, rainfall, density, and time. Biol. $511 \quad$ Conserv. $137,461-472$.

512 Githaka, N., Konnai, S., Bishop, R., Odongo, D., Lekolool, I., Kariuki, E., Gakuya, F., Kamau, L., 513 Isezaki, M., Murata, S., Ohashi, K., 2014. Identification and sequence characterization of novel 514 515 516 517 518 519 Theileria genotypes from the waterbuck (Kobus defassa) in a Theileria parva-endemic area in Kenya. Vet. Parasitol. 202, 180-193.

Gortazar, C., Ferroglio, E., Hofle, U., Frolich, K., Vicente, J., 2007. Diseases shared between wildlife and livestock: a European perspective. Eur. J. Wildl. Res. 53, 241-256.

Grootenhuis, J.G., 2000. Wildlife, livestock and animal disease reservoirs, In: Wildlife Conservation By Sustainable Use. Springer, pp. 81-113.

Gubbels, J.M., de Vos, A.P., van der Weide, M., Viseras, J., Schouls, L.M., de Vries, E., Jongejan, F., 1999. Simultaneous detection of bovine Theileria and Babesia species by reverse line blot hybridization. J. Clin. Microbiol. 37, 1782-1789.

Guindon, S., Dufayard, J.-F., Lefort, V., Anisimova, M., Hordijk, W., Gascuel, O., 2010. New algorithms and methods to estimate maximum-likelihood phylogenies: assessing the performance of PhyML 3.0. Syst. Biol. 59, 307-321.

Guindon, S., Gascuel, O., 2003. A simple, fast, and accurate algorithm to estimate large phylogenies by maximum likelihood. Syst. Biol. 52, 696-704.

Harvey, J.W., Simpson, C.F., Gaskin, J.M., 1978. Cyclic thrombocytopenia induced by a Rickettsia-like agent in dogs. J. Infect. Dis. 137, 182-188. 
Heyman, P., Cochez, C., Hofhuis, A., van der Giessen, J., Sprong, H., Porter, S.R., Losson, B., Saegerman, C., Donoso-Mantke, O., Niedrig, M., Papa, A., 2010. A clear and present danger: tick-borne diseases in Europe. Expert Rev. Anti Infect. Ther. 8, 33-50.

Hooge, J., Howe, L., Ezenwa, V.O., 2015. Identification of novel Theileria genotypes from Grant's gazelle. Int. J. Parasitol. Parasites Wildl. 4, 239-243.

Jongejan, F., Uilenberg, G., 2004. The global importance of ticks. Parasitol. 129, S3-S14.

Kawahara, M., Rikihisa, Y., Lin, Q., Isogai, E., Tahara, K., Itagaki, A., Hiramitsu, Y., Tajima, T., 2006. Novel genetic variants of Anaplasma phagocytophilum, Anaplasma bovis, Anaplasma centrale, and a novel Ehrlichia sp. in wild deer and ticks on two major islands in Japan. Appl. Environ. Microbiol. 72, 1102-1109.

Kimura, M., 1980. A simple method for estimating evolutionary rates of base substitutions through comparative studies of nucleotide sequences. J. Molec. Evol. 16, 111-120.

Kinnaird, M.F., O'Brien, T.G., 2012. Effects of private-land use, livestock management, and human tolerance on diversity, distribution, and abundance of large African mammals. Conserv. Biol. 26, 1026-1039.

Kock, R. 2005. What is this infamous wildlife/livestock disease interface? A review of current knowledge for the African continent. In IUCN Species Survival Commission Report on Conservation and Development Interventions at the Wildlife/Livestock Interface Implications for Wildlife, Livestock and Human Health, S, O., ed., pp. 1-14.

Latif, A.A., Hove, T., Kanhai, G.K., Masaka, S., 2002. Buffalo-associated Theileria parva: the risk to cattle of buffalo translocation into the Highveld of Zimbabwe. Ann. N. Y. Acad. Sci. 969, 275279. 
Li, Y., Chen, Z., Liu, Z., Liu, J., Yang, J., Li, Q., Li, Y., Ren, Q., Niu, Q., Guan, G., Luo, J., Yin, H., 2014. First report of Theileria and Anaplasma in the Mongolian gazelle, Procapra gutturosa. Parasit. Vectors 7, 614.

Liu, Z., Ma, M., Wang, Z., Wang, J., Peng, Y., Li, Y., Guan, G., Luo, J., Yin, H., 2012. Molecular survey and genetic identification of Anaplasma species in goats from Central and Southern China. Appl. Environ. Microbiol. 78, 464-470.

Maxwell, J.F., Lea, B.-F., Davies, T.J., 2013. The study of parasite sharing for surveillance of zoonotic diseases. Environ. Res. Lett. 8, 015036.

McCoy, K.D., Léger, E., Dietrich, M., 2013. Host specialization in ticks and transmission of tick-borne diseases: a review. Front. Cell. Infect. Microbiol. 3, 57.

Miller, R.S., Farnsworth, M.L., Malmberg, J.L., 2013. Diseases at the livestock-wildlife interface: Status, challenges, and opportunities in the United States. Prev. Vet. Med. 110, 119-132.

Nishi, J.S., Stephen, C., Elkin, B.T., 2002. Implications of agricultural and wildlife policy on management and eradication of bovine tuberculosis and brucellosis in free-ranging wood bison of Northern Canada. Ann. N. Y. Acad. Sci. 969, 236-244.

Njiiri, N.E., Bronsvoort, B.M.d., Collins, N.E., Steyn, H.C., Troskie, M., Vorster, I., Thumbi, S.M., Sibeko, K.P., Jennings, A., van Wyk, I.C., Mbole-Kariuki, M., Kiara, H., Poole, E.J., Hanotte, O., Coetzer, K., Oosthuizen, M.C., Woolhouse, M., Toye, P., 2015. The epidemiology of tickborne haemoparasites as determined by the reverse line blot hybridization assay in an intensively studied cohort of calves in western Kenya. Vet. Parasitol. 210, 69-76.

Nylander, J., 2004. MrModeltest v2. Program distributed by the author. Evolutionary Biology Centre, Uppsala University 2. 
574 Olwoch, J.M., Reyers, B., Engelbrecht, F.A., Erasmus, B.F.N., 2008. Climate change and the tick-borne disease, Theileriosis (East Coast fever) in sub-Saharan Africa. J. Arid Environ. 72, 108-120.

R-Development-Team 2015. R: A Language and Environment for Statistical Computing. In R Foundation for Statistical Computing (Vienna Austria R Foundation for Statistical Computing.).

Schnittger, L., Yin, H., Qi, B., Gubbels, M.J., Beyer, D., Niemann, S., Jongejan, F., Ahmed, J.S., 2003. Simultaneous detection and differentiation of Theileria and Babesia parasites infecting small ruminants by reverse line blotting. Parasitol. Res. 92, 189-196.

Shaw, S.E., Day, M.J., Birtles, R.J., Breitschwerdt, E.B., 2001. Tick-borne infectious diseases of dogs. Trends Parasitol. 74-80.

Siembieda, J.L., Kock, R.A., McCracken, T.A., Newman, S.H., 2011. The role of wildlife in transboundary animal diseases. Anim. Health Res. Rev. 12, 95-111.

Swofford, D.L., 2003. PAUP*. Phylogenetic Analysis Using Parsimony (* and Other Methods). Version 4.

Talavera, G., Castresana, J., 2007. Improvement of phylogenies after removing divergent and ambiguously aligned blocks from protein sequence alignments. Syst. Biol. 56, 564-577.

Tamura, K., Nei, M., 1993. Estimation of the number of nucleotide substitutions in the control region of mitochondrial DNA in humans and chimpanzees. Molec. Biol. Evol. 10, 512-526.

Tamura, K., Stecher, G., Peterson, D., Filipski, A., Kumar, S., 2013. MEGA6: Molecular Evolutionary Genetics Analysis Version 6.0. Molec. Biol. Evol. 30, 2725-2729.

Uilenberg, G., 1995. International collaborative research: significance of tick-borne hemoparasitic diseases to world animal health. Vet. Parasitol. 57, 19-41. 
595 Walker, A.R., Bouattour, A., Camicas, J., Estrada-Pena, A., Horak, I., Latif, A., Pegram, R., Preston, P., 596 2003. Ticks of domestic animals in Africa: a guide to identification of species. Bioscience 597 Reports, Edinburgh, $221 \mathrm{pp}$.

598 Wiethoelter, A.K., Beltrán-Alcrudo, D., Kock, R., Mor, S.M., 2015. Global trends in infectious diseases 599 at the wildlife-livestock interface. Proc. Nat. Acad. Sci. U. S. A. 112, 9662-9667.

600 Yabsley, M.J., McKibben, J., Macpherson, C.N., Cattan, P.F., Cherry, N.A., Hegarty, B.C., 601 Breitschwerdt, E.B., O’Connor, T., Chandrashekar, R., Paterson, T., Perea, M.L., Ball, G., 602 Friesen, S., Goedde, J., Henderson, B., Sylvester, W., 2008. Prevalence of Ehrlichia canis, 603 Anaplasma platys, Babesia canis vogeli, Hepatozoon canis, Bartonella vinsonii berkhoffii, and 604 Rickettsia spp. in dogs from Grenada. Vet. Parasitol. 151, 279-285.

605 Yang, J., Li, Y., Liu, Z., Liu, J., Niu, Q., Ren, Q., Chen, Z., Guan, G., Luo, J., Yin, H., 2015. Molecular 606 detection and characterization of Anaplasma spp. in sheep and cattle from Xinjiang, northwest 607 China. Parasit. Vectors 8, 1-7.

608 Zobba, R., Anfossi, A.G., Pinna Parpaglia, M.L., Dore, G.M., Chessa, B., Spezzigu, A., 2014. Molecular 609 investigation and phylogeny of Anaplasma spp. in Mediterranean ruminants reveal the presence 610 of neutrophil-tropic strains closely related to A. platys. Appl. Environ. Microbiol. 80, 271-280. 